\title{
Is there evidence that we should screen the general population for Lynch syndrome with genetic testing? A systematic review
}

This article was published in the following Dove Press journal:

Pharmacogenomics and Personalized Medicine

20 February 2017

Number of times this article has been viewed

\author{
Anya E R Prince' \\ $R$ Jean Cadigan ${ }^{1,2}$ \\ Gail E Henderson ${ }^{1,2}$ \\ James P Evans ${ }^{1,3-6}$ \\ Michael Adams ${ }^{1,3}$ \\ Emmanuel \\ Coker-Schwimmer ${ }^{7}$ \\ Dolly C Penn² \\ Marcia Van Riper 1,8 \\ Giselle Corbie-Smith ${ }^{1,2,6}$ \\ Daniel E Jonas ${ }^{1,6,7}$ \\ 'Center for Genomics and Society, \\ ${ }^{2}$ Department of Social Medicine, \\ ${ }^{3}$ Department of Genetics, ${ }^{4}$ Carolina \\ Center for Genome Sciences, \\ ${ }^{5}$ Lineberger Comprehensive Cancer \\ Center, ${ }^{6}$ Department of Medicine, \\ ${ }^{7}$ Cecil G. Sheps Center for Health \\ Services Research, ${ }^{8}$ School of Nursing, \\ The University of North Carolina- \\ Chapel Hill, Chapel Hill, NC, USA
}

Correspondence: Emmanue

Coker-Schwimmer

Cecil G. Sheps Center for Health

Services Research, 725 Martin Luther

King Jr. Blvd., CB 7590, Chapel Hill,

NC 27599-7590, USA

Tel + I 9198436692

Email maschwim@email.unc.edu
Background: The emerging dual imperatives of personalized medicine and technologic advances make population screening for preventable conditions resulting from genetic alterations a realistic possibility. Lynch syndrome is a potential screening target due to its prevalence, penetrance, and the availability of well-established, preventive interventions. However, while population screening may lower incidence of preventable conditions, implementation without evidence may lead to unintentional harms. We examined the literature to determine whether evidence exists that screening for Lynch-associated mismatch repair (MMR) gene mutations leads to improved overall survival, cancer-specific survival, or quality of life. Documenting evidence and gaps is critical to implementing genomic approaches in public health and guiding future research.

Materials and methods: Our 2014-2015 systematic review identified studies comparing screening with no screening in the general population, and controlled studies assessing analytic validity of targeted next-generation sequencing, and benefits or harms of interventions or screening. We conducted meta-analyses for the association between early or more frequent colonoscopies and health outcomes.

Results: Twelve studies met our eligibility criteria. No adequate evidence directly addressed the main question or the harms of screening in the general population. Meta-analyses found relative reductions of $68 \%$ for colorectal cancer incidence (relative risk: $0.32,95 \%$ confidence interval: $0.23-0.43$, three cohort studies, 590 participants) and $78 \%$ for all-cause mortality (relative risk: $0.22,95 \%$ confidence interval: $0.09-0.56$, three cohort studies, 590 participants) for early or more frequent colonoscopies among family members of people with cancer who also had an associated MMR gene mutation.

Conclusion: Inadequate evidence exists examining harms and benefits of population-based screening for Lynch syndrome. Lack of evidence highlights the need for data that directly compare benefits and harms.

Keywords: Lynch syndrome, systematic review, targeted next-generation sequencing, genetic screening, general population

\section{Introduction}

Advances in DNA sequencing and the gradual emergence of preventive interventions for certain genetic diseases have spurred growing interest in population-based genomic screening as a possible public health intervention. ${ }^{1,2}$ Recent calls to screen women in the general US population for $B R C A 1 / 2$ mutations are an example. ${ }^{3}$ It is presumed that such screening could minimize morbidity and mortality related to preventable conditions by identifying individuals who harbor unidentified genetic mutations that lead to a high risk for these conditions. Indeed, despite the absence of 
systematic investigation of the harms and benefits associated with genetic screening in asymptomatic populations, several groups and commercial efforts are already beginning to screen the general adult population for genetic mutations. ${ }^{4-7}$ Lynch syndrome is often included in such screening programs because of its nontrivial prevalence and high penetrance (50\%-80\% of family members of those diagnosed with cancer who carry such mutations will develop colorectal cancer [CRC] without preventive measures), and because interventions with well-documented efficacy are available. Lynch syndrome, also called hereditary nonpolyposis CRC, is associated with an increased risk of multiple cancers, including colorectal, endometrial, ovarian, stomach, small intestine, and skin. ${ }^{8}$ Mutations in the MMR genes $(M L H 1$, MSH2, MSH6, and PMS2) associated with Lynch syndrome occur in an estimated one out of every 440 people and are associated with a range of penetrance estimates (generated from studying family members of MMR mutation carriers diagnosed with cancer): $50 \%-80 \%$ risk of colon cancer and $40 \%-60 \%$ risk of endometrial cancer. ${ }^{8-10}$ Clinical modalities, such as frequent screening colonoscopies to remove polyps, gynecological screenings, and prophylactic surgery, are currently used and considered effective preventive measures for people with a personal or family history of Lynch-associated cancers and MMR gene mutations. ${ }^{10-12}$

To date, relevant systematic reviews have focused on screening individuals at high risk due to a family or personal medical history of cancer, but have not examined the evidence for net benefits of screening asymptomatic adults. ${ }^{12,13}$ For example, in 2009, the Center for Disease Control's Evaluation of Genomic Applications in Practice and Prevention (EGAPP) Working Group completed a systematic review of the net benefits of performing molecular screening of tumors for evidence of Lynch syndrome in individuals newly diagnosed with CRC. They found sufficient evidence to recommend tumor screening, given the potential benefits for identifying at-risk relatives through cascade germline testing, but the focus on newly diagnosed cancer patients leaves unanswered questions for the larger asymptomatic adult population. ${ }^{12}$ While there is robust literature and evidence about the benefits of screening symptomatic populations, an asymptomatic population may experience different harms or benefits and may have different penetrance rates than those with a personal or family history of cancer. Before implementing population screening efforts, it is important to understand the evidence of harms and benefits specific to the target population.
Therefore, we conducted a systematic review to evaluate the evidence on benefits and harms associated with screening for Lynch syndrome in the general adult population using targeted next-generation sequencing. We included evidence related to any of the four MMR genes associated with Lynch syndrome. Although specific genes vary in their precise impact on risk (eg, variations exist with regard to the penetrance of CRC and uterine cancer in those with different Lynch-associated gene mutations), we included all four genes in our analyses to increase the breadth and likelihood of identifying relevant studies. Our review is particularly timely due to recent advances in DNA sequencing technology that have driven interest in such screening in the absence of data about benefits and harms. Our overarching question was whether there is direct evidence that screening asymptomatic adults for MMR gene mutations with modern sequencing modalities (ie, massively parallel, also known as "next-generation", sequencing) leads to improved overall survival, cancer-specific survival, or quality of life.

\section{Materials and methods}

We developed an analytic framework and five accompanying questions (Supplementary material 1) to guide the systematic review following procedures used by the US Preventive Services Task Force. ${ }^{14}$ The analytic framework included five questions. First, our overarching question: Is there direct evidence that screening asymptomatic adults with genetic testing for MMR gene mutations ( $M L H 1, M S H 2, P M S 2$, and MSH6) leads to improved overall survival, cancer-specific survival, or quality of life? Second, what is the accuracy and reliability of targeted next-generation sequencing compared with Sanger sequencing and deletion/duplication testing for detecting mutations in MMR genes in asymptomatic adults? Third, for asymptomatic adults with an MMR gene mutation, and their family members who elect to be tested, does genetic counseling and early introduction of cancer-specific preventive measures reduce the incidence of cancers compared with routine cancer screening? Fourth, do counseling and early detection/preventive measures/interventions improve overall survival, cancer-specific survival, or quality of life for individuals and their family members who test positive for an MMR gene mutation? Fifth, are harms associated with genetic screening for MMR gene mutations using targeted next-generation sequencing or with subsequent interventions?

We present findings from each of these five questions in our results. Anticipating that we were unlikely to find any 
primary studies directly addressing our overarching question (ie, randomized controlled trials [RCTs] comparing screening with no screening and enrolling adults from the general population), our analytic framework constructed an indirect pathway potentially linking evidence on screening to health outcomes.

\section{Data sources and searches}

In May 2014, an experienced evidence-based practice center librarian searched PubMed, EMBASE, CINAHL, and the Cochrane Library for English-language papers published from June 2006 through May 2014 (Table S1, in Supplementary material 2). The 2006 date corresponds to the timing of last searches conducted by other reviews and technology assessments, especially the 2009 EGAPP review. ${ }^{14,15}$ To identify relevant papers published prior to June 2006, we manually searched reference lists of pertinent reviews and studies.

\section{Study selection}

All abstracts and full-text articles were independently reviewed by at least two investigators using prespecified eligibility criteria (Table S2, in Supplementary material 2), with disagreements resolved by group discussion. For our overarching question, we searched for RCTs comparing screening with no screening in asymptomatic adults without previous or current diagnosis of Lynch-associated cancers; however, non-RCTs, cohort studies, and modeling studies were also eligible. Given our anticipation of a limited number of studies of asymptomatic adults without a personal or family history of Lynch syndrome or associated cancers, studies of asymptomatic individuals with a family history were also eligible for inclusion.

\section{Data extraction and risk of bias assessment}

For each paper, one investigator identified and extracted relevant data using structured data extraction forms, while a second reviewed for completeness and accuracy. To assess studies' risk of bias, two independent reviewers used predefined criteria based on established guidance. ${ }^{16-18}$ We rated the studies as low, medium, high, or unclear risk of bias. ${ }^{17}$ For studies addressing analytic validity, we assessed risk of bias using the QUADAS-2. ${ }^{19}$ Disagreements were resolved through group discussion. Tables S3-S14 (in Supplementary material 2) provide details of risk of bias assessment criteria and outcomes. Data extraction and assessment occurred between May 2014 and November 2015.

\section{Data synthesis and analysis}

We qualitatively synthesized findings for each question by summarizing the characteristics and results of included studies in tabular and narrative format. Meta-analyses were conducted using Stata ${ }^{\circledR}$ version 11 (Stata Press, College Station, TX, USA) when we identified three or more studies making the same comparison and reporting similar outcomes. We used random effects models with the inverse-variance weighted method (DerSimonian and Laird) to estimate relative risks (RRs). ${ }^{20}$ For all quantitative syntheses, the $\chi^{2}$ and $I^{2}$ statistics were calculated to assess statistical heterogeneity in effects between studies..$^{21,22}$

We graded the strength of evidence as high, moderate, low, or insufficient based on an established approach that incorporates four key domains: risk of bias, consistency, directness, and precision. ${ }^{23,24}$ Tables S15-S23 (in Supplementary material 2) detail our strength of evidence assessments, which were determined by group consensus.

\section{Results}

Of 2,147 abstracts and 120 full papers reviewed, 12 studies met our eligibility criteria (Figure 1) - one modeling study addressed our overarching question; ${ }^{25}$ two studies addressed analytic validity; ${ }^{26,27}$ six studies addressed the impact of early or more frequent colonoscopies ${ }^{28-30}$ or gynecological screenings or prophylactic surgery ${ }^{31-33}$ on cancer incidence or survival; and five studies addressed harms of screening and interventions..$^{30,31,34-36}$ Table 1 describes the characteristics and outcomes of each of our included studies.

Included studies, other than those examining analytic validity, used prospective cohort, ${ }^{29,30}$ retrospective cohort, ${ }^{28,31-33}$ and modeling designs. ${ }^{25,34-36}$ No included studies were controlled trials. Both analytic validity studies were cross-sectional comparisons between next-generation and Sanger sequencing. ${ }^{26,27}$ We found no eligible studies reporting on quality of life, overdiagnosis, false-positive test results, disease-specific distress or anxiety, burden of responsibility associated with communicating positive test results with family, false-negative test results, or loss of insurance or inability to be insured.

\section{Question I: overarching question}

The only included study for our overarching question was a cost-effectiveness analysis with a simulation framework integrating models of colorectal and endometrial cancers with a five-generation family history model to predict health and economic outcomes. ${ }^{25}$ The study modeled 20 primary screening strategies for a simulated population of 100,000 


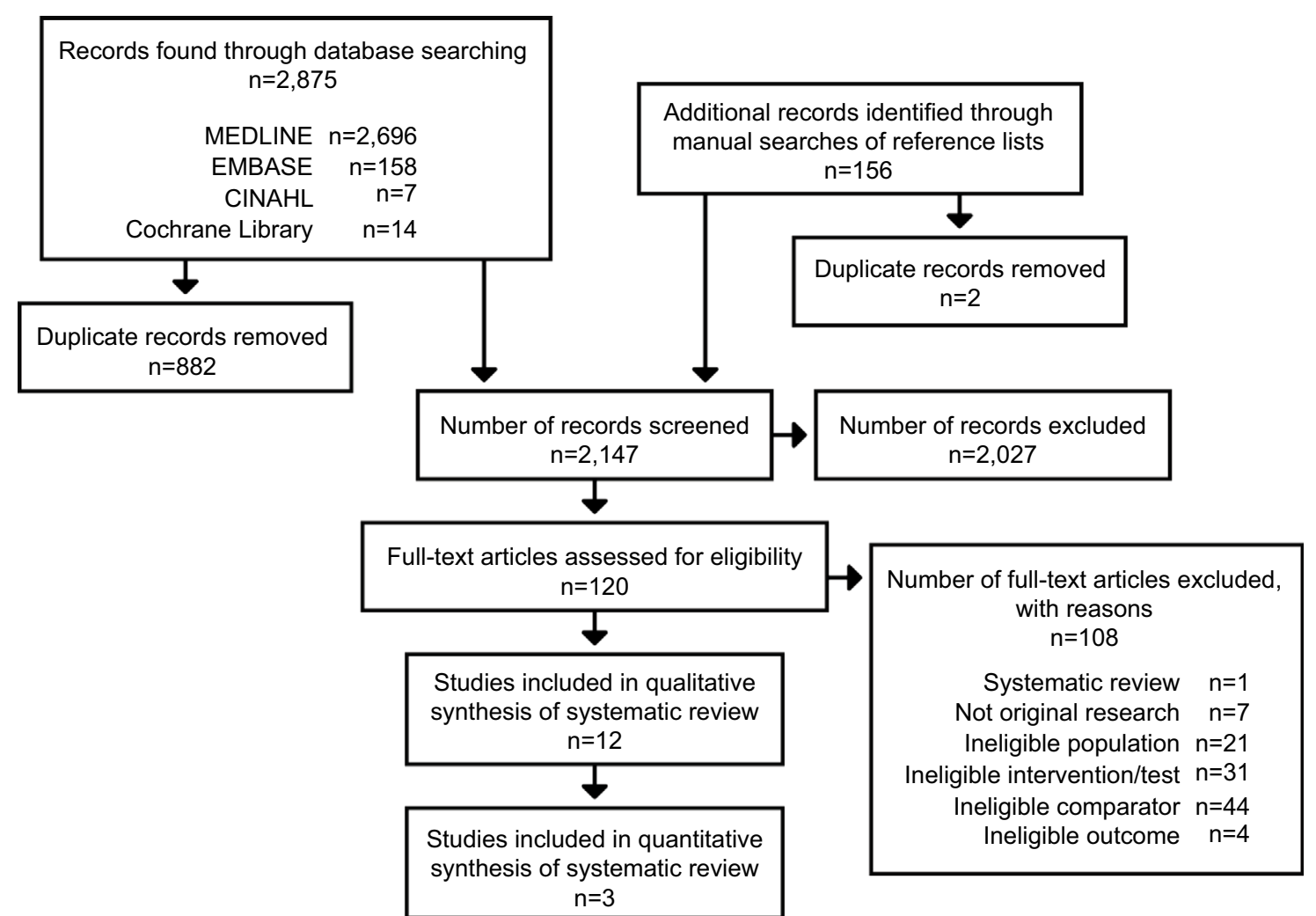

Figure I Summary of searches and study selection.

Notes: This figure is a flowchart that presents the yield of the review's literature retrieval process and the results of each stage of literature review, including title and abstract review, and full-text article review. A total of 2,875 records were retrieved through electronic database searches, including MEDLINE, EMBASE, CINAHL, and the Cochrane Library, and 156 records from additional records identified through manual searches of reference lists. After removal of 884 duplicate records, a total of 2,147 titles and abstracts were screened, and 2,027 of these were excluded. Next, 120 full-text articles were reviewed for eligibility. Of these, 108 articles were excluded because of using a systematic review design with incompatible eligibility criteria, not being original research, ineligible population, ineligible interventions/tests, ineligible comparators, and ineligible outcomes. The final yield included in the systematic review was 12 studies reported in 12 papers. Of these, three studies provided data that could be quantitatively synthesized in meta-analyses.

people representative of the US population. The 20 screening strategies started at different ages $(20,25,30,35$, or 40 years) and different thresholds for risk of carrying one of the four MMR mutations (based on PREMM ${ }_{126}$ model: $0 \%$, $2.5 \%, 5 \%$, or $10 \%) .{ }^{37}$

The 20 hypothetical screening strategies yielded anywhere from 0.41 to 4.07 life-years saved per carrier exposed to screening; a strategy of universal screening starting at age 20 resulted in the greatest gain. However, universal screening (using no risk threshold) was not cost-effective. As the model increased the risk threshold for genetic testing, fewer people received primary genetic screening, and qualityadjusted life-years (QALYs) gained per 100,000 simulated individuals decreased while cost-effectiveness steadily improved (Table 1).

Some study strengths included using the validated Archimedes model and some inputs from a systematic review conducted for EGAPP. ${ }^{15}$ Key limitations of the study included the lack of available inputs from the general asymptomatic population, using a risk prediction model developed from and validated for people referred for genetic testing and individuals with CRC (rather than the general population), limited use of sensitivity analyses to explore the potential impact of variation in uncertain inputs, not including other cancers for the probands (besides colorectal or endometrial) that occur more frequently in people with Lynch syndrome, and omission of indirect costs. In light of these limitations, many of which bias the results in favor of the intervention, we rated the study as high risk of bias for the clinical effectiveness assessment of universal screening and for all of the models using the PREMM risk prediction (Table S3. in Supplementary material 2). However, we rated the risk of bias as medium for the universal screening cost-effectiveness assessment (noting that the intervention was not found to be cost-effective). We graded the strength of evidence as insufficient (Table S15, in Supplementary material 2).

\section{Question 2: analytic validity}

Two studies met eligibility criteria for our analytic validity question. ${ }^{26,27}$ Both compared the performance of 


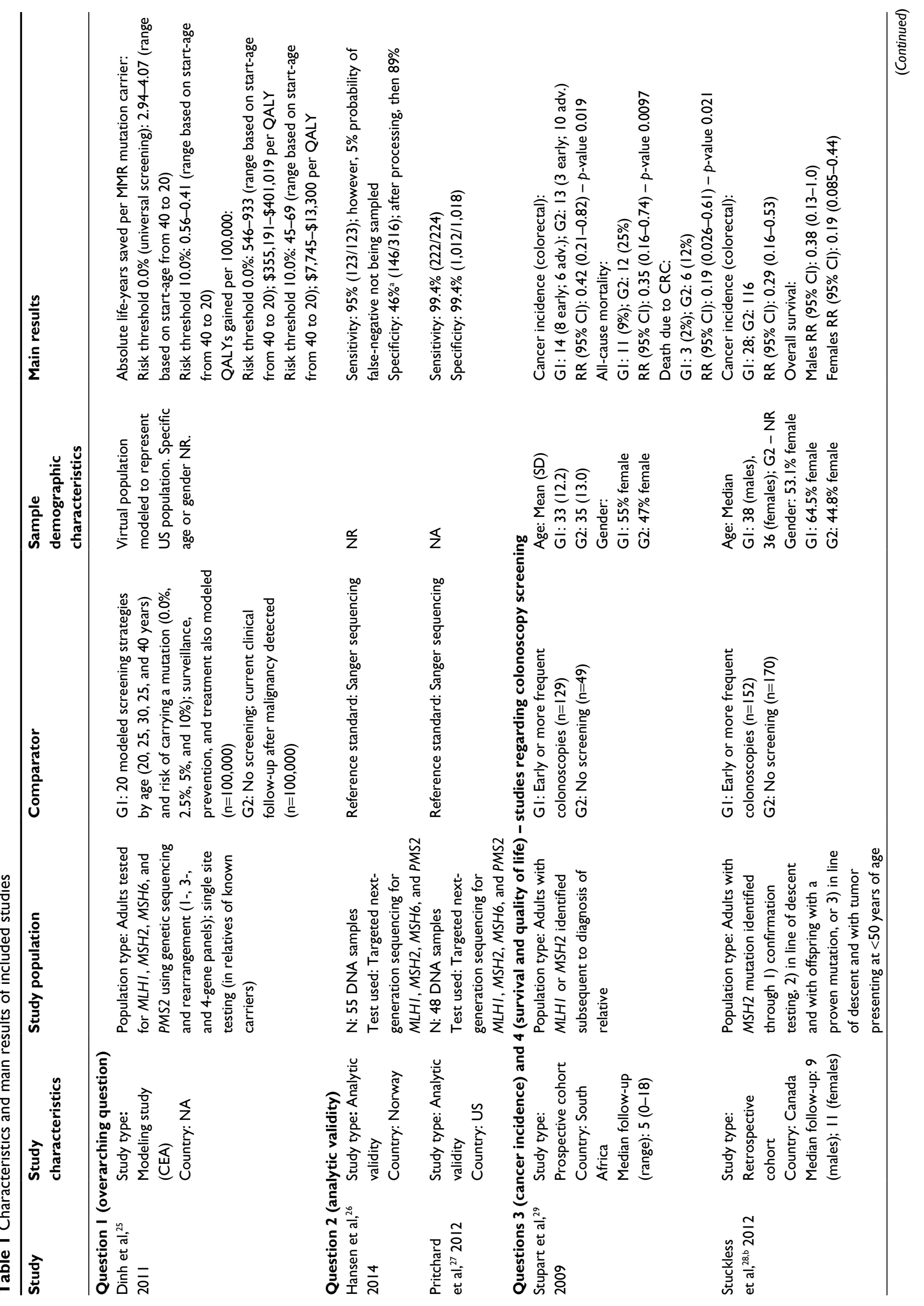




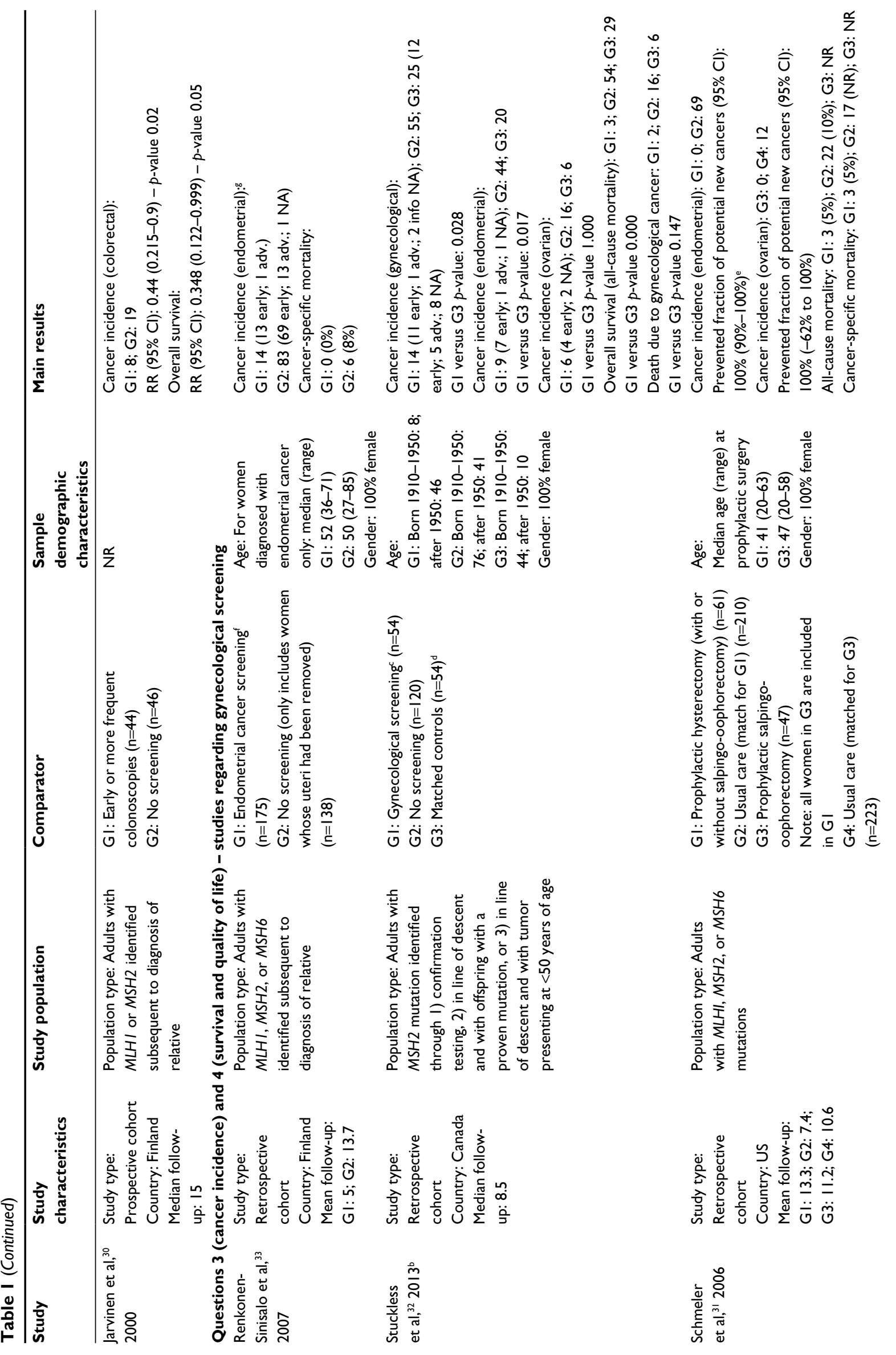



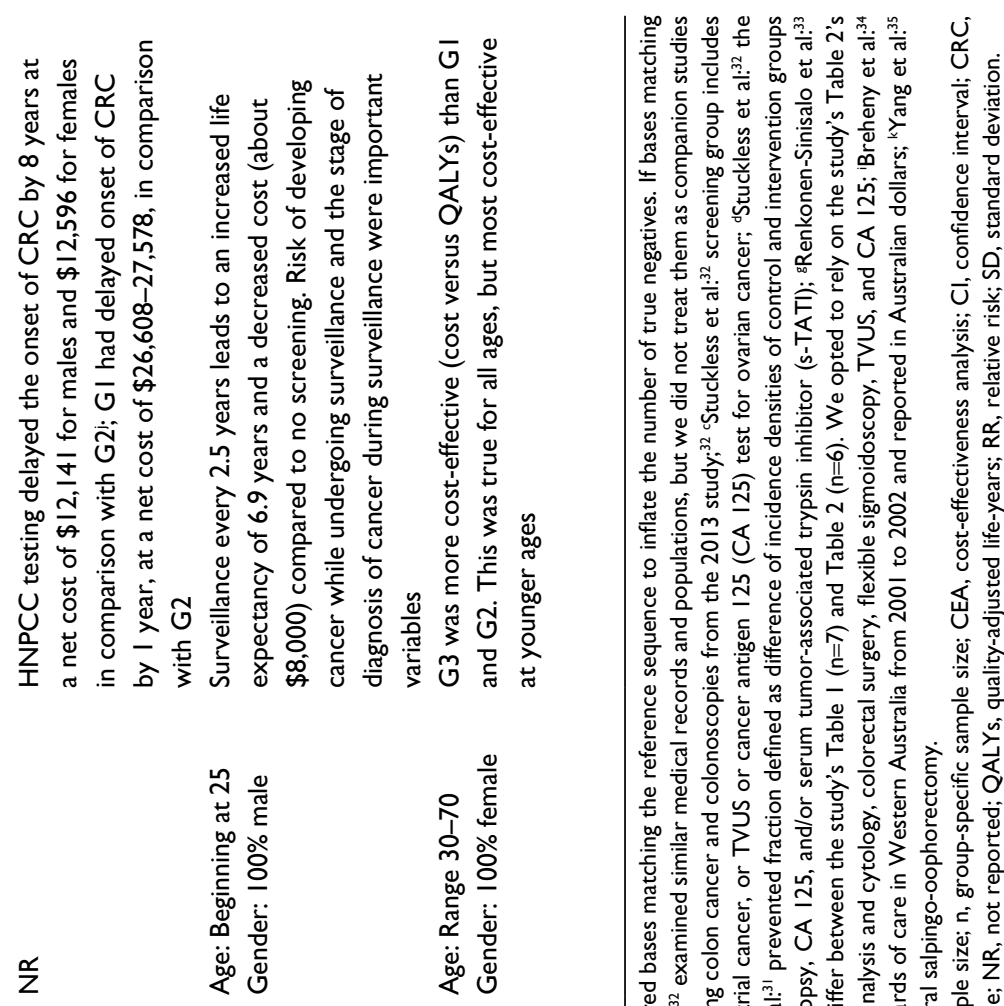

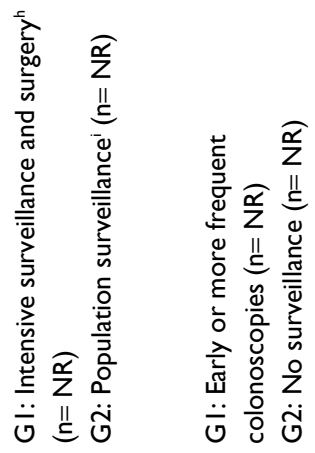
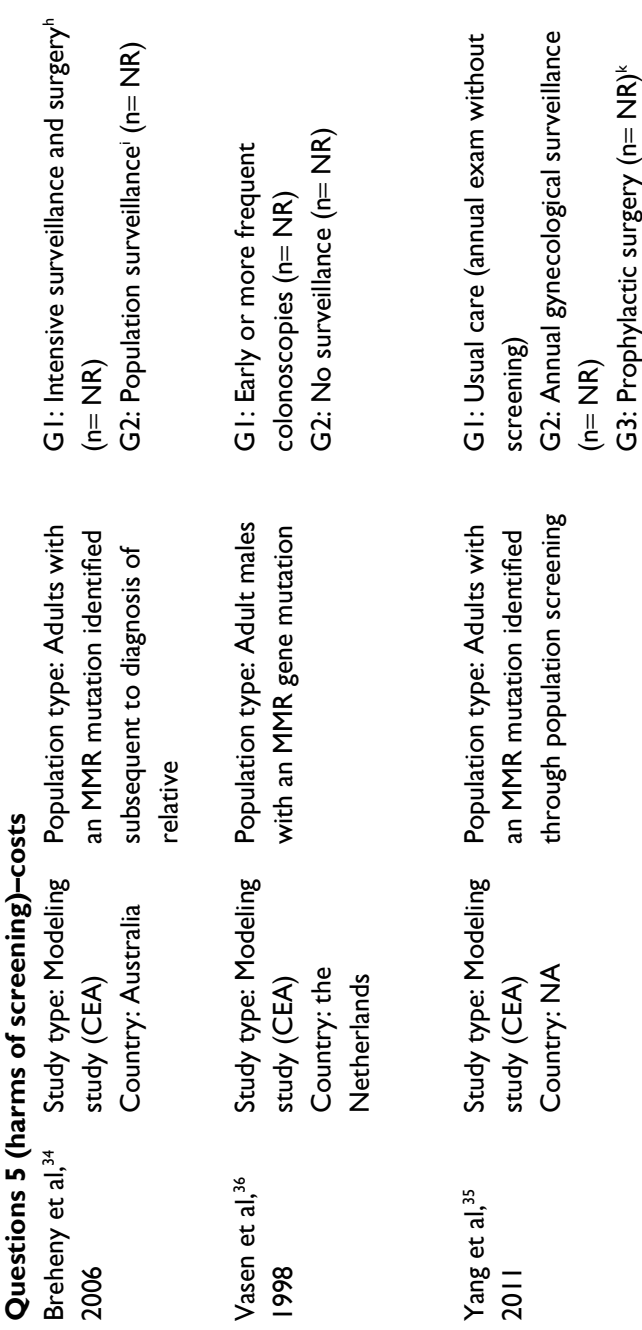

焉

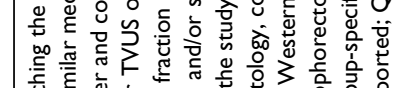

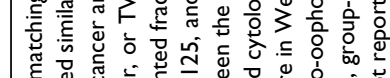

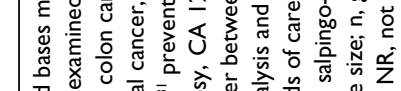

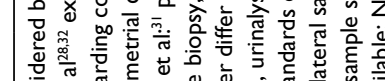

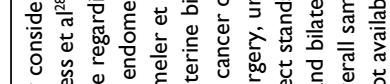

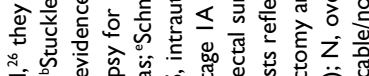

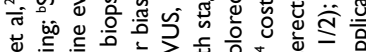

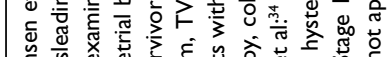

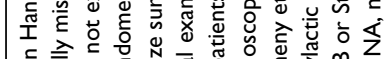

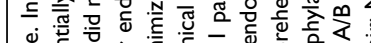

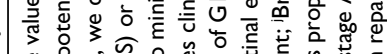

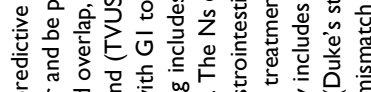

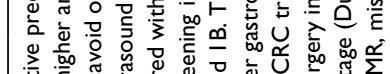

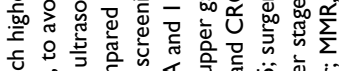

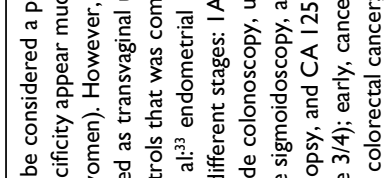

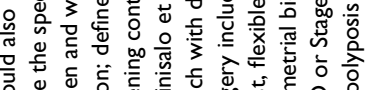

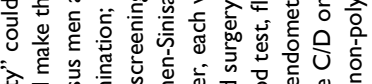

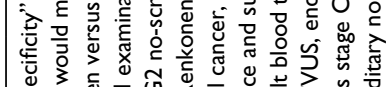

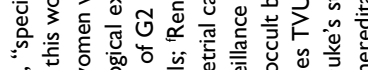

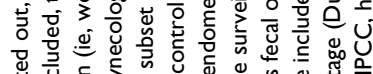

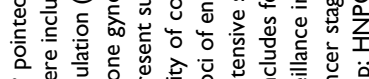

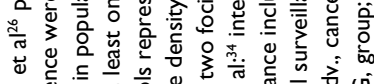

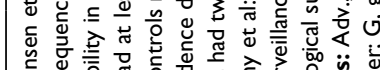

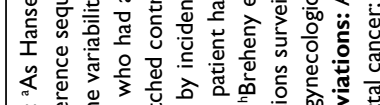

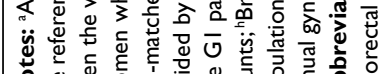

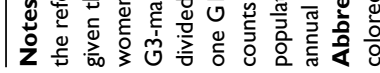


next-generation sequencing technologies with traditional Sanger sequencing (the reference standard). Hansen et $\mathrm{al}^{26}$ tested 55 DNA samples for deleterious variants in the four MMR mutations, 39 of which were previously known to carry a deleterious mutation. Sixteen of the samples were previously uncharacterized, only evaluated as a test of the workflow, and did not contribute to characterization of the sensitivity/specificity of next-generation sequencing as a diagnostic test. Pritchard et $\mathrm{al}^{27}$ tested 82 samples for mutations in genes classically implicated in Lynch syndrome. We extracted data on samples relevant to our review to calculate sensitivity and thus included the following: 23 from patients with known mutations in Lynch-associated genes or other familial colon cancers, and six public samples from a sequencing consortium. Nineteen samples from patients without any family history of cancer were evaluated for purposes of addressing specificity (Table 1).

Both studies reported high sensitivity and specificity for next-generation sequencing of Lynch-associated genes. The included studies reported sensitivities and specificities of $95 \%-100 \%$ and $89 \%-99.4 \%$, respectively (Table 1 ). We rated both studies as low risk of bias (Tables S4 and S5, in Supplementary material 2) and graded the strength of evidence as high for both sensitivity and specificity (Tables S16 and S17, in Supplementary material 2).

\section{Questions 3 and 4: cancer incidence and survival}

\section{Early or more frequent colonoscopy}

Three studies examined the potential benefits of early or more frequent colonoscopies (with polypectomy, as needed) for adult family members with an MMR gene mutation (Table 1). Participants were identified either through testing subsequent to a first-degree relative's cancer diagnosis ${ }^{29,30}$ or through confirmation mutation testing, being in the line of descent and having offspring with a proven mutation, or being in the line of descent and presenting clinically with a Lynch-associated tumor before age $50 .{ }^{28}$ The included controls were individuals who had an identified MMR gene mutation, ${ }^{29,30}$ or who were identified or presumed to have a mutation based on familial and clinical indications ${ }^{28}$ and failed to undergo recommended colonoscopy screening. Two studies tested for both $M L H 1$ and MSH2; ${ }^{29,30}$ one included only MSH2 ${ }^{28}$ Two were prospective cohort studies, ${ }^{29,30}$ and one was a retrospective cohort. ${ }^{28}$ All three reported $\mathrm{CRC}$ incidence and overall survival, and one reported cancer-specific survival. ${ }^{29}$

Overall, the three studies reported lower rates of CRC for those undergoing colonoscopy and polypectomy than for the control group. Our meta-analysis found a relative reduction of almost 70\% in CRC incidence (RR: $0.32,95 \%$ confidence interval [CI]: 0.23-0.43, $I^{2}:$ 7.7\%) (Supplementary material 3 ). Additionally, Stupart et $\mathrm{al}^{29}$ reported that cancers diagnosed in individuals who underwent colonoscopies were more likely to be early stage (Duke's A/B) than late stage (Duke's $\mathrm{C} / \mathrm{D})$, and late-stage cancers were more likely in unscreened individuals (Table 1).

Our meta-analysis showed that early or more frequent colonoscopies were associated with a significant reduction in all-cause mortality (RR: $0.22,95 \%$ CI: $0.09-0.56 ; I^{2}: 77.0 \%$ ) (Supplementary material 4). Although each study found a statistically significant reduction in all-cause mortality, the analysis revealed considerable statistical heterogeneity. Our analysis, stratified by study design, identified differences in design as the possible cause of the heterogeneity, with the retrospective cohort study ${ }^{28}$ finding a greater effect size than the prospective studies. One study reported cancer-specific survival and found $\mathrm{CRC}$ to be the cause of death for $2 \%$ of those undergoing colonoscopies as compared with $12 \%$ of controls (RR: 0.19, 95\% CI: 0.026-0.61) (Table 1). ${ }^{29} \mathrm{We}$ rated the risk of bias as low, ${ }^{29}$ medium, ${ }^{30}$ and high (due to risk of selection and survivor biases) ${ }^{28}$ (Tables S7, S10, and $\underline{\mathrm{S} 12}$, in Supplementary material 2). We graded the strength of evidence of the effects of colonoscopy on both CRC incidence and overall survival as moderate and the strength of evidence for cancer-specific survival as low (Tables S18, $\underline{\mathrm{S} 21}$, and S22, respectively, in Supplementary material 2).

\section{Gynecological screening and/or prophylactic surgery}

We included three retrospective cohort studies. Two studies examined incidence of and survival from gynecological cancers in adult family members with an MMR gene mutation who had undergone gynecological screening. ${ }^{32,33} \mathrm{~A}$ third study examined the effect of prophylactic gynecological surgery in women with an MMR mutation identified from hereditary-cancer registries. ${ }^{31}$ Specific screening and surgical interventions examined varied for each study (Table 1). Controls were individuals with identified MMR mutations ${ }^{31,33}$ or who were known or presumed to have a mutation based on familial and clinical indications ${ }^{32}$ and who did not undergo gynecological screening ${ }^{32,33}$ or surgery. ${ }^{31}$ Two studies examined $M L H I, M S H 2$, and $M S H 6$ carriers; ${ }^{31,33}$ one included only MSH2 carriers. ${ }^{32}$ All three studies examined cancer survival and incidence of endometrial cancer; two also examined incidence of ovarian cancer. ${ }^{31,32}$

Two studies found that gynecological screening had little effect on cancer incidence or survival, while the third 
study found prophylactic surgery to be effective ${ }^{31}$ (Table 1). Schmeler et $\mathrm{al}^{31}$ found that no woman who had undergone prophylactic hysterectomy subsequently had endometrial cancer, compared with $33 \%$ of the control group's women (prevented fraction of potential new cancers: $100 \%, 95 \% \mathrm{CI}$ : 90\%-100\%). Similarly, no one who had undergone bilateral salpingo-oophorectomy at the time of their hysterectomy was subsequently diagnosed with ovarian cancer, whereas $5 \%$ of the control group's women developed ovarian cancer (prevented fraction of potential new cancers: 100\%, 95\% CI: $-62 \%-100 \%)$. Three participants in the surgery group died (from colon, brain, and bladder cancer, respectively), while 22 died in the control group. Of these 22, 17 died from various cancers, one from cardiac disease, and four from causes unknown.

We rated the study of prophylactic surgery ${ }^{31}$ as having a medium risk of bias, and the two papers on gynecological screening as having high risk of bias ${ }^{32,33}$ (due to selection bias and risk of confounding). The strength of evidence ratings for endometrial cancer and ovarian cancer incidence were low and insufficient, respectively, for both gynecological screening and surgery (Tables S19 and S20, in Supplementary material 2). Additionally, we graded the strength of evidence of both cancer-specific and overall survival following screenings or surgery as insufficient (Tables S21 and S22. in Supplementary material 2).

\section{Question 5: harms of screening and interventions \\ Complications due to surgery}

One study reported the death of a control group patient due to a pulmonary embolism after a successful colectomy. ${ }^{30}$ Another study reported complications of ureteral injury, ureterovaginal fistula, and ureteroenteral fistula in a woman who had prophylactic abdominal hysterectomy with salpingooophorectomy and a prior history of rectal carcinoma treated by rectosigmoid resection with colostomy and creation of a Hartmann's pouch. ${ }^{31}$

\section{Costs}

Three modeling studies presented somewhat relevant data on costs of preventive interventions for people with Lynch syndrome or testing relatives of people known to have Lynch syndrome (Table 1). Yang et al ${ }^{35}$ modeled cost-effectiveness for a theoretical population of women with Lynch syndrome at age 30, comparing prophylactic surgery (hysterectomy and bilateral salpingo-oophorectomy) with two forms of surveillance (annual gynecological screening and annual gynecological exam), to predict endometrial or ovarian cancer outcomes. ${ }^{35}$ The authors found that risk-reducing surgery led to the lowest costs and highest number of QALYs (\$23,224 per patient for 25.71 QALYs for surgery, compared with \$68,392 for 25.17 QALYs for annual screening, and $\$ 100,484$ for 24.60 QALYs for annual exam). A second study by Breheny et $\mathrm{al}^{34}$ presented a decision analysis for asymptomatic first-degree relatives of known Lynch mutation carriers. ${ }^{34}$ Using life expectancy and costs of surveillance and surgery in Western Australia for individuals aged 25-70 years, they found a net savings and one CRC-free year gained when comparing relatives who are tested to relatives who are not tested but have increased colonoscopy and gynecological surveillance. However, when comparing testing to a control group with population surveillance only, they found eight CRC-free years gained at an overall net cost. ${ }^{34}$ In the final study, Vasen et $\mathrm{al}^{36}$ modeled the costeffectiveness of increased colonoscopy surveillance for male Lynch syndrome mutation carriers. ${ }^{36}$ They found cost savings and a 7-year increased life expectancy for those receiving surveillance versus no surveillance. We graded the strength of evidence of these studies as insufficient given the medium risk of bias, the reliance on various evidence sources and assumptions, and the lack of reporting on precision estimates (Table S23, in Supplementary material 2).

\section{Discussion}

Overall, we found little evidence to inform an assessment of the harms and benefits of screening asymptomatic adults without a personal or family history of Lynch-associated cancers for Lynch syndrome with targeted next-generation sequencing. The vast majority of papers exploring the effectiveness of interventions studied individuals already diagnosed with cancer or their family members. Of the 12 included studies, three were modeling studies and two examined analytic validity. The remaining seven examined the effects of early or more frequent colonoscopies or gynecological screenings and surgeries on cancer incidence and mortality among individuals already diagnosed with cancer or their family members. The 12 studies focused on Lynchassociated colorectal and gynecological cancers. In spite of the fact that Lynch syndrome, like many genetic conditions, is pleiotropic, which raises unique concerns regarding returning results and explaining implications and medical interventions, ${ }^{38}$ we found no evidence about screening measures for other Lynch-associated cancers, such as skin cancers, hepatobiliary cancer or transitional cell carcinomas of the renal pelvis or ureter. 
In population-based screening programs, asymptomatic individuals face potential harms that are distinct from those faced by individuals with symptoms and their relatives, such as overdiagnosis (identifying mutations that would never have caused a problem) leading to anxiety and unnecessary treatment, or misinterpreting the meaning of negative results for Lynch-associated mutations (giving false reassurance that routine CRC screening is unnecessary for them). ${ }^{39}$ Further complicating population-based interventions, current penetrance estimates for Lynch-associated mutations (and other Mendelian conditions) are based on families with a high incidence of the disorder in question; penetrance of mutations found in the general population is likely lower than that estimated in published studies. Despite these potential differences in harms for asymptomatic individuals, we found an absence of evidence on harms of screening the general population for Lynch syndrome. Our initial inclusion/exclusion criteria comprised a broad range of potential harms, from overdiagnosis and overtreatment to misinterpretation of negative or uncertain results and increased cost (Table S2, in Supplementary material 2). Despite the breadth of our search, we found only three modeling studies examining cost and two studies that briefly described complications postintervention.

Misinterpretation of results and subsequent overtreatment is a major potential harm of genomic screening of an asymptomatic population. Any time individuals undergo genetic sequencing, numerous variants of uncertain significance are generated. In screening asymptomatic populations, the probability that any given variant of uncertain significance represents an actual deleterious Lynch variant is very low. This reality necessitates an extremely rigorous variant classification and reporting scheme to avoid reporting a large number of false positives that would commit a vast number of individuals to unnecessary surveillance. ${ }^{40}$

\section{Limitations}

We did not include uncontrolled studies that evaluated the benefits or harms of relevant interventions. To be eligible, studies were required to have a comparator group. The studies included in our review were not designed or adequately powered to assess complications of colonoscopy or other interventions. Although we found limited evidence that met our inclusion criteria, other bodies of literature have described harms from the preventive interventions in our review. For example, serious harms including perforations, hemorrhage, diverticulitis, cardiovascular events, severe abdominal pain, and death are estimated to occur in 2.8 per 1,000 screening colonoscopies (95\% CI: $1.5-5.2$ per 1,000 procedures $){ }^{41}$ Furthermore, we identified a number of noncomparative studies addressing psychosocial harms and the short- and long-term impacts of predictive testing that did not meet our eligibility criteria. ${ }^{42-44}$ Most authors, however, acknowledge methodological limitations in these studies as well as selection biases inherent in self-referred, motivated study populations.

\section{Conclusion}

In summary, there are increasing calls to implement genomic screening at the population level, and Lynch syndrome is an appealing candidate for such programs. Overall, however, our systematic review shows that there is inadequate evidence examining the potential harms and benefits of such population-based screening. The need for rigorous investigation is especially compelling at present given the large uncontrolled experiment that is currently being conducted by multiple groups, including commercial efforts, which are embarking upon such screening without a sufficient underlying evidence base. Some research projects are beginning to explore the impact of genomic testing in asymptomatic populations without a personal or family history of cancer, but clearly more research is needed before we can begin to understand the implications of how genomic screening may differ from testing in symptomatic patients and their relatives, and what benefits and harms it may entail when broadly applied to asymptomatic populations.

\section{Acknowledgments}

Research reported in this publication was supported by the National Institutes of Health through the National Human Genome Research Institute under Award Numbers P50HG004488 (all authors, except DP) and U01HG006487 (GEH and JPE), the National Heart, Lung, and Blood Institute under Award Number HL105493 (GC-S), and the US Department of Health and Human Services through the Health Resources and Services Administration under Award Number 5-D33-HP25771 (DP). The content is solely the responsibility of the authors and does not necessarily represent the official views of the National Institutes of Health. All authors participated in the conception and design of the systematic review, the analysis of the data, and the drafting and revising of the manuscript. The review's literature searches were conducted by Christiane Voisin, MLS. Additional data analysis support was received from 
Roberta Wines, Gabriel Lázaro-Muñoz, Alexi McHugh, and Malika Roman Isler. We also thank the two anonymous reviewers for their helpful comments and suggestions to improve this manuscript.

\section{Disclosure}

The authors report no other conflicts of interest in this work.

\section{References}

1. Evans JP, Berg JS, Olshan AF, Magnuson T, Rimer BK. We screen newborns, don't we?: realizing the promise of public health genomics. Genet Med. 2013;15(5):332-334.

2. Guttmacher AE, Collins FS, Khoury MJ, McCabe LL, McCabe ER. Population screening in the age of genomic medicine. $N$ Engl J Med. 2003;348(1):50-58.

3. Levy-Lahad E, Lahad A, King MC. Precision medicine meets public health: population screening for BRCA1 and BRCA2. J Natl Cancer Inst. 2015;107(1):420.

4. Million Veteran Program (MVP). Available from: http://www.research. va.gov/mvp/. Accessed November 11, 2015.

5. The 100,000 Genomes Project. Available from: http://www.genomicsengland.co.uk/about-genomics-england/. Accessed November 4, 2015.

6. Ray T. Companies Begin Subsidizing Color Genomics' Breast, Ovarian Cancer Testing for Employees. GenomeWeb; 2015. Available from: https://www.genomeweb.com/molecular-diagnostics/companies-beginsubsidizing-color-genomics-breast-ovarian-cancer-testing. Accessed December 2, 2016.

7. Helger M. Geisinger Begins Returning Clinically Actionable Exome Sequencing Results to Patients. GenomeWeb; 2015.

8. Kohlmann W, Gruber SB. Lynch syndrome. In: Pagon RA, Adam MP, Ardinger HH, et al., editors. GeneReviews ${ }^{\circledR}$. Seattle, WA: University of Washington; 1993.

9. Chen S, Wang W, Lee S, et al. Prediction of germline mutations and cancer risk in the Lynch syndrome. JAMA. 2006;296(12):1479-1487.

10. Lindor NM, Petersen GM, Hadley DW, et al. Recommendations for the care of individuals with an inherited predisposition to Lynch syndrome: a systematic review. JAMA. 2006;296(12):1507-1517.

11. Vasen HF, Blanco I, Aktan-Collan K, et al. Revised guidelines for the clinical management of Lynch syndrome (HNPCC): recommendations by a group of European experts. Gut. 2013;62(6):812-823.

12. Recommendations from the EGAPP Working Group: genetic testing strategies in newly diagnosed individuals with colorectal cancer aimed at reducing morbidity and mortality from Lynch syndrome in relatives. Genet Med. 2009;11(1):35-41.

13. Moyer VA. Risk assessment, genetic counseling, and genetic testing for BRCA-related cancer in women: US Preventive Services Task Force recommendation statement. Ann Intern Med. 2014;160(4):271-281.

14. US Preventive Services Task Force Procedure Manual. Available from: https://www.uspreventiveservicestaskforce.org/Page/Name/methodsand-processes?. Accessed December 2, 2016.

15. Palomaki GE, McClain MR, Melillo S, Hampel HL, Thibodeau SN. EGAPP supplementary evidence review: DNA testing strategies aimed at reducing morbidity and mortality from Lynch syndrome. Genet Med. 2009;11(1):42-65.

16. Agency for Healthcare Research and Quality. Methods Guide for Effectiveness and Comparative Effectiveness Reviews. Rockville, MD: Agency for Healthcare Research and Quality; 2014.

17. Viswanathan M, Ansari MT, Berkman ND, et al. Assessing the Risk of Bias of Individual Studies in Systematic Reviews of Health Care Interventions. Rockville, MD: AHRQ Publication No. 12-EHC047-EF; 2012.

18. Agency for Healthcare Research and Quality. Methods Guide for Medical Test Reviews. Rockville, MD: Agency for Healthcare Research and Quality; 2012.
19. Whiting PF, Rutjes AW, Westwood ME, et al. QUADAS-2: a revised tool for the quality assessment of diagnostic accuracy studies. Ann Intern Med. 2011;155(8):529-536.

20. Sutton AJ, Abrams KR, Jones DR, et al. Methods for Meta-Analysis in Medical Research (Wiley Series in Probability and Statistics-Applied Probability and Statistics Section). London, UK: Wiley; 2000.

21. Higgins JP, Thompson SG. Quantifying heterogeneity in a meta-analysis. Stat Med. 2002;21(11):1539-1558.

22. Higgins JP, Thompson SG, Deeks JJ, Altman DG. Measuring inconsistency in meta-analyses. BMJ. 2003;327(7414):557.

23. Owens DK, Lohr KN, Atkins D, et al. AHRQ series paper 5: grading the strength of a body of evidence when comparing medical interventions - Agency for Healthcare Research and Quality and the Effective Health-Care Program. J Clin Epidemiol. 2010;63(5):513-523.

24. Berkman ND, Lohr KN, Ansari MT, et al. Grading the strength of a body of evidence when assessing health care interventions: an EPC update. J Clin Epidemiol. 2014;68(11)1312-1324.

25. Dinh TA, Rosner BI, Atwood JC, et al. Health benefits and costeffectiveness of primary genetic screening for Lynch syndrome in the general population. Cancer Prev Res (Phila). 2011;4(1):9-22.

26. Hansen MF, Neckmann U, Lavik LA, et al. A massive parallel sequencing workflow for diagnostic genetic testing of mismatch repair genes. Mol Genet Gen Med. 2014;2(2):186-200.

27. Pritchard CC, Smith C, Salipante SJ, et al. ColoSeq provides comprehensive lynch and polyposis syndrome mutational analysis using massively parallel sequencing. J Mol Diagn. 2012;14(4):357-366.

28. Stuckless S, Green JS, Morgenstern M, et al. Impact of colonoscopic screening in male and female Lynch syndrome carriers with an MSH2 mutation. Clin Gen. 2012;82(5):439-445.

29. Stupart DA, Goldberg PA, Algar U, Ramesar R. Surveillance colonoscopy improves survival in a cohort of subjects with a single mismatch repair gene mutation. Colorectal Dis. 2009;11(2):126-130.

30. Jarvinen HJ, Aarnio M, Mustonen H, et al. Controlled 15-year trial on screening for colorectal cancer in families with hereditary nonpolyposis colorectal cancer. Gastroenterology. 2000;118(5):829-834.

31. Schmeler KM, Lynch HT, Chen LM, et al. Prophylactic surgery to reduce the risk of gynecologic cancers in the Lynch syndrome. $N$ Engl J Med. 2006;354(3):261-269.

32. Stuckless S, Green J, Dawson L, et al. Impact of gynecological screening in Lynch syndrome carriers with an MSH2 mutation. Clin Gen. 2013;83(4):359-364.

33. Renkonen-Sinisalo L, Butzow R, Leminen A, Lehtovirta P, Mecklin JP, Jarvinen HJ. Surveillance for endometrial cancer in hereditary nonpolyposis colorectal cancer syndrome. Int J Cancer. 2007;120(4):821-824.

34. Breheny N, Geelhoed E, Goldblatt J, Ee H, O'Leary P. Economic evaluation of the familial cancer programme in Western Australia: predictive genetic testing for familial adenomatous polyposis and hereditary nonpolyposis colorectal carcinoma. Community Genet. 2006;9(2):98-106.

35. Yang KY, Caughey AB, Little SE, Cheung MK, Chen LM. A costeffectiveness analysis of prophylactic surgery versus gynecologic surveillance for women from hereditary non-polyposis colorectal cancer (HNPCC) families. Fam Cancer. 2011;10(3):535-543.

36. Vasen HF, van Ballegooijen M, Buskens E, et al. A cost-effectiveness analysis of colorectal screening of hereditary nonpolyposis colorectal carcinoma gene carriers. Cancer. 1998;82(9):1632-1637.

37. Kastrinos F, Steyerberg EW, Mercado R, et al. The PREMM(1,2,6) model predicts risk of MLH1, MSH2, and MSH6 germline mutations based on cancer history. Gastroenterology. 2011;140(1):73-81.

38. Kocarnik JM, Fullerton SM. Returning pleiotropic results from genetic testing to patients and research participants. JAMA. 2014;311(8): 795-796.

39. Prince AE, Berg JS, Evans JP, Jonas DE, Henderson G. Genomic screening of the general adult population: key concepts for assessing net benefit with systematic evidence reviews. Genet Med. 2015;17(6):441-443.

40. Adams MC, Evans JP, Henderson GE, Berg JS, Investigators G. The promise and peril of genomic screening in the general population. Genet Med. 2016;18(6):593-599. 
41. Whitlock EP, Lin JS, Liles E, Beil TL, Fu R. Screening for colorectal cancer: a targeted, updated systematic review for the US Preventive Services Task Force. Ann Intern Med. 2008;149(9):638-658.

42. Bleiker EM, Esplen MJ, Meiser B, Petersen HV, Patenaude AF. 100 years Lynch syndrome: what have we learned about psychosocial issues? Fam Cancer. 2013;12(2):325-339.
43. Sie AS, Prins JB, Spruijt L, Kets CM, Hoogerbrugge N. Can we test for hereditary cancer at 18 years when we start surveillance at 25? Patient reported outcomes. Fam Cancer. 2013;12(4):675-682.

44. Collins VR, Meiser B, Ukoumunne OC, Gaff C, St John DJ, Halliday JL. The impact of predictive genetic testing for hereditary nonpolyposis colorectal cancer: three years after testing. Genet Med. 2007;9(5):290-297.

\section{Publish your work in this journal}

Pharmacogenomics and Personalized Medicine is an international, peerreviewed, open access journal characterizing the influence of genotype on pharmacology leading to the development of personalized treatment programs and individualized drug selection for improved safety, efficacy and sustainability. This journal is indexed on the American Chemical
Society's Chemical Abstracts Service (CAS). The manuscript management system is completely online and includes a very quick and fair peer-review system, which is all easy to use. Visit http://www.dovepress. com/testimonials.php to read real quotes from published authors. 